\title{
La Geografía en Cataluña: el problema de las comarcas
}

\author{
Adela Gil Crespo
}

\section{INTRODUCCIÓN}

La lectura de las obras de Pau Vila publicadas por la editorial Curial, la de la Geografía de Cataluña, dirigida por Solé Sabaris, la Revista Catalana de Geografía, la Conferencia de Casassas i Simó en la Real Sociedad Geográfica, me han decidido a escribir sobre la Geografía en Cataluña, que tiene unas características particulares. La búsqueda de la identidad catalana ha hecho que desde el pasado siglo geógrafos, historiadores, etnólogos, literatos hayan buscado en la tierra, en los archivos, en las tradiciones populares, las características específicas de esta porción de la Península lbérica.

Hoy que tan de moda está entre los geógrafos el estudio del espacio, para contribuir con los planificadores a ordenarle racionalmente, o, en colaboración con los ambientalistas, para frenar su deterioro, en $\mathrm{Ca}$ taluña, por razones político-administrativas, el espacio catalán viene siendo objeto de minuciosos estudios por parte de geógrafos, naturalistas, antropólogos, juristas, etc.

La Geografía ha desempeñado un importante papel como trataré de dar a conocer en esta breve exposición, ya que el intentar establecer unas demarcaciones que respondan a las características del pueblo catalán y a sus necesidades, presupone el conocimiento en profundidad del terreno y de los hombres que lo ocupan. Considerando el papel de la Geografía expondré, haciendo uso de las publicaciones citadas: el nacimiento de la Geografía catalana, el excursionismo y la Geografía, la Geo- 
grafía en la escuela, la obra de Pau Vila, y en particular los problemas territoriales de la demarcación comarcal; las comarcas establecidas en la Segunda República, las comarcas de los geógrafos en la Geografía de Cataluña, y el porqué de la división comarcal y no provincial.

\section{NACIMIENTO DE LA GEOGRAFÍA CATALANA}

No voy a referirme a la Cartografía medieval, sino a las inquietudes científicas en el siglo XVIII, período que pese al cierre de Universidades con el Decreto de Nueva Planta (1715) y la creación de la Universidad teológica de Cervera, dice Solé Sabaris " "dio nacimiento, paradógicamente, a buen número de los intelectuales que tenian que impulsar el movimiento literario y romántico de la Renaixança, inspirado en el culto a su tierra y a sus perdidas libertades".

Por necesidades de relaciones comerciales, al abrirse el comercio de Cataluña con América, en tiempos de Carlos III se sintió la necesidad de adquirir conocimientos científicos al margen de las prohibiciones reales. Nacida en 1764, en la Academia de Ciencias de Barcelona se impartirán enseñanzas de química, botánica, matemáticas, Geografía, etc.

Con el desarrollo que alcanzarian las Ciencias Naturales la Geografía que era una ciencia descriptiva (como lo fue durante largos años) se enriquecerá. Una pléyade de hombres de ciencia, citados por Solé Sabaris, a lo largo del siglo $x i x$ se esforzaran en inventariar los recursos y en buscar las raíces de la personalidad catalana. Cabe citar algunos nombres, Francisco de Bolos (1775-1844), descubridor de la región volcánica de Olot; Pedro Alsius (1839-1915), descubridor de la mandíbula de Bañolas y las grandes figuras de la geología catalana Luis Marian Vidal (1842-1922) y el canónigo Jaime Almera y Comas (1845-1919).

Creo que en Cataluña, como en Madrid, es a través de las Ciencias Naturales, conocimiento directo del terreno, como llevaran a cabo los hombres de la Institución y del Museo de Ciencias Naturales, como se llegará a la Geografía y a la etnología. Simo).

' Solé Sabaris, Los primeros geólogos catalanes (tomado del prólogo de Casassas i 
Alcanzó, al finalizar el siglo xIX y comienzos del actual, una particular importancia el excursionismo. Dice Casassas que en Cataluña los hombres de la Renaixança pusieron un énfasis especial en el excursionismo, no con un fin deportivo sino con una viva intencionalidad política y patriótica. Así nacieron las asociaciones; Catalanista de Excursiones Científicas, el Club Muntanyenc, la Sociedad de Ciencias Naturales, figurando entre sus socios Pi Sunyer, Bosch, Guimpera y más tarde figuraría en el club Muntanyenc, el gran geólogo Llopin Lladó.

Al comenzar el siglo, en el Centro Excursionista de Cataluña el geólogo y geógrafo Font i Sagué, al que se le debe el estudio científico de las comarcas de Cataluña, creó una sección de Geología y Geografía física, de gran concurrencia y prestigio. Es en esta asociación en la que nacerían los primeros geógrafos modernos de Cataluña. La Geografía tendrá un marcado carácter científico, pues se impartirá, ya al finalizar el siglo xix y comienzos del actual en la Facultad de Ciencias. No es exclusivo de Cataluña, pues sucederá lo mismo en el Centro; la Geografía física va unida a los trabajos de los naturalistas, recordemos a los Hernández Pacheco, Dantin Cereceda, etc. En Cataluña figurarán los nombres de Jaime Almera, J. R. Bataller, José Broquetas, Font y Sagué Salvaña, etc.

La escuela desempeñará un papel importante en la introducción de la Geografía moderna en Cataluña. Dice Casassas "gran número de maestros, entre ellos los renovadores de la nueva Geografía catalana, J. Palau Vera, Miguel Santaló, Pau Vila, Pedro Blasi, etc., dieron un gran impulso a los estudios geográficos y su actuación coetanea marca de una manera decisiva el curso posterior de la Geografía en Cataluña y hay que tener presente el papel de los maestros, en la divulgación de nuevos conceptos geográficos, tanto individualmente como en el marco de las instituciones surgidas del movimiento de renovación pedagógica catalana, que tuvo lugar en las primeras décadas del siglo XX».

En la renovación, o mejor dicho en la creación de la Geografía catalana, con su profunda preocupación por el espacio y su ordenación, se ha de destacar la figura de Pau Vila. A su obra y a su personalidad le han dedicado estudios, entre otros el profesor Casassas. Perteneció como otros ilustres geógrafos catalanes a Magisterio. A principios de siglo en 1906, se creó la escuela de Magisterio en Barcelona, que introdujo una importante renovación pedagógica, en particular en el estudio de la Geografía, con el estudio de la Ciencias Naturales y las excursiones geográficas con los alumnos. 
Pau Vila introducirá en Cataluña las tendencias de la escuela francesa, el estudio regional y comarcal de Vidal de la Blache y Gallois. Tomó parte activa en el movimiento excursionista, siendo nombrado en el año 1931 presidente del Centro Excursionista de Cataluña. Al plantearse el problema de la división administrativa de Cataluña, en la Segunda República, con el Estatuto Catalán formó parte de la Ponencia para la división comarcal de Cataluña, de la que más adelante hablaré.

El fin de la Guerra Civil le llevó al destierro, y en las tierras americanas siguió su labor de geógrafo e investigador, en el Gimnasio Moderno de Bogotá y en el Instituto Pedagógico de Caracas. A su regreso a España continuó su labor de geógrafo, recojo las palabras de Casassas «él como los mejores, sintió la terrible e incesante necesidad de ir descubriendo el país y descubierto, formarlo".

Parte de su obra se ha publicado en la editorial Curial, que recojo en la Bibliografía.

\section{LA ORDENACIÓN DEL ESPACIO CATALÁN}

Existen dos momentos en la historia de Cataluña en las que se pretende borrar y uniformar la personalidad de esta región. Un momento es la aplicación del Decreto de Nueva Planta de 1715, otro en el siglo $X I X$, con la división político-administrativa, en provincias de 1833. Ambos tuvieron como consecuencia en diferentes momentos históricos, «el reconocimiento de la unidad catalana y el derecho de los catalanes a ordenar su territorio en la forma que considerasen conveniente". De este deseo, nacerá la necesidad de conocer bien el territorio, de conocer a sus gentes, formas de vida, tradiciones. Bien es verdad, que no es sólo una inquietud catalana, es la que en Francia llevó a Vidal de la Blache y a sus discípulos al estudio regional, la que en Portugal llevó a Leite de Vasconcelos, padre de la etnología portuguesa a recoger y estudiar las tradiciones del país; en Galicia a Otero Pedraio, a recorrer su tierra y dejarnos la Geografía de Galicia, en Madrid a los hombres de la Institución a recorrer la Sierra y la Meseta.

Pero volviendo a Cataluña, la división territorial del pasado siglo, rompió la unidad, como en otras regiones de España, borrando en gran parte las divisiones tradicionales de las comarcas, y será la preocupación por buscar su identidad y delimitación, lo que lleve a geógrafos e historia- 
dores a establecer la ordenación comarcal del espacio. Así dice Casassas «moltes vegades s'ha assenyalat que aqueste cami de la geografia catalana vers els problemes de l'espai té precedents ben llunyanos. Es pode citar, i la llista no es completa, els noms de Calça, Pere Gil, Onofre Manescal, Mateu Aimeric, i tantos d'altres que, des de mitjan segle XVIII formen l'aplec de los precursors" ?

La inquietud por la comarcalización llevaría desde el pasado siglo hasta el primer tercio del actual a los geógrafos y naturalistas a plantearse problemas sobre lo contenido en una comarca y en su delimitación. Irán apareciendo en libros escolares, como los de Pedro Blasi, la Geografía de Catalunya, y en la Geografía escolar de Catalunya, la división y estudio comarcal, en las obras de Miguel Santaló con el estudio de Cataluña y el estudio de la comarca.

Dice Casassas que, con la introducción del posibilismo vidaliano, y la formulación racional y científica del nacionalismo político, el estudio comarcal tomará una nueva orientación, figurando los nombres del historiador Rovira i Virgili, $\mathrm{Pi}$ i Sunyer, Valls i Taberner, Bofill i Mates, y los geógrafos José Iglesies y Pau Vila.

\section{EL MOVIMIENTO COMARCALISTA Y EL PROYECTO DE DIVISIÓN EN COMARCAS}

Con la llegada de la Segunda República, se acentuará el movimiento comarcalista. Se procederá al estudio histórico de las diferentes divisiones territoriales de Cataluña. Pau Vila, en un ensayo recogerá las divisiones históricas, protohistóricas, romanas, visigodas, las divisiones medievales en veguerias, los corregimientos filipistas y las divisiones territoriales del siglo XIX, exponiendo en su ensayo los origenes del movimiento comarcalista, del que procuraré extractar las ideas principales.

Pau Vila dice en su ensayo que la palabra "comarca" no aparece en los escritores clásicos, en el sentido de expresar una entidad territorial; ya entrado el siglo XVII, algunos escritores al describir Cataluña, dan el nombre de comarcas, apareciendo los nombres populares del Ampur-

\footnotetext{
2 Prólogo de la Divisió Territorial de Catalunya, p. 17.
} 
dan, Gironès, Selva, Vallés, Barcelonés, Penedés, Camp. de Tarragona. Algunos nombres señoriales como el conado de Prades, priorato de Scala Dei, o administrativas como la vegueria de Tortosa.

En el mismo siglo, Andreu Bosch ${ }^{3}$ dice que «toda la tierra se divide en 49 partes", sobreentendiéndose que son las comarcas. Pedro Gil, en la Historia natural y eclesiástica de Cataluña dice que toda Cataluña puede dividirse en veintisiete partes, climas o comarcas o parte de tierra. José Aparici, en el siglo xVIII, en su obra Descripción y Planta del Principado de Cataluña, habla de la división en obispados y veguerías, señalando que la demarcación más conocida es la de comarcas, citando el número de treinta.

Pero será en el siglo XIX y primer tercio del XX, como se ha dicho, cuando el movimiento comarcalista toma fuerza y cuando se considera que «la comarca es la base territorial de gobierno". "En la Asamblea de Unión Catalanista de Manresa de 1892" se dice textualmente: “La divisió territorial sobre la cual es desensotlla la gradació jerárquica tindrá per fonament la comarca natural, el municipi» (Vila, 1977: 67). «En el año 1897 Esteve Sunyol en una conferencia del Ateneo Barcelonés fijó de una manera concreta la división comarcal de Cataluña con una base político-administrativa. Más tarde en 1928, el movimiento separatista, en la Asamblea celebrada en La Habana bajo la presidencia de Macia, al redactar la Constitución provisional de la República de Cataluña, se dividía al país en comarcas como organismos territoriales de gobierno".

\section{GEOGRAFIA Y COMARCAS}

Al desarrollo de la geografía, con nuevos principios de investigación, producirá en el primer tercio de nuestro siglo una serie de monografías comarcales. Respondian a lo que en Francia habia hecho Gallois (Regions naturelles et noms de pays), y lo que harán en Madrid, los naturalistas Dantin Cereceda y Hernández Pacheco, y lo que la Real Sociedad Geográfica había patrocinado, desde los primeros años de su existencia.

${ }^{3}$ P. VILA. La divisió territorial de Catalunya, p. 48. 
Los historiadores habian manifestado vivo interés en las demarcaciones históricas de Cataluña, pero la aparición de estudios de carácter científico, como el Vallés del geólogo Font y Sagué, unida a la influencia francesa darán lugar a nuevos plantamientos. Así Santaló dirá "que la comarca es una unidad territorial de estructura geológica similar en la que relieve, clima, vegetación, y la acción humana actuan en reciprocidad".

El problema se planteará a la hora de delimitar, pues si bien hay un núcleo, que diríamos central, en el que las características de individualización se presentan con nitidez, no sucede lo mismo en las perifericas comarcales en las que aparecen zonas de transición, diluyéndose los caracteres específicos.

Entre las monografías que irán apareciendo, obra de geógrafos serán: El Gironés de Miguel Santaló y Parvorell, estudio de una comarca, que apareciendo en citas, no se presentaba en la cartografía catalana. Era un estudio de enfoque moderno en el que para su delimitación y contenido debían entrar en juego los factores naturales y los humanos.

Uno de los problemas claves del estudio era el de la delimitación, en este sentido en 1930, apareció la monografía del geógrafo y excursionista José Iglesies Delimitació del Camp de Tarragona. Decidido partidario de la comarca natural procedió a un meticuloso estudio de los factores naturales y la incidencia de las relaciones humanas, procediendo a la demarcación de las comarcas limítrofes. Haciendo uso de la historia, presentó en su estudio las diferentes demarcaciones históricas del Campo de Tarragona en las comarcas limítrofes. Factores naturales y factores históricos hay que tener presentes en los estudios comarcales. El mismo autor haría otro estudio sobre el Priorato.

Se han de sumar a estos estudios comarcales, los de Gonzalo de Reparaz, hijo, sobre la Plana de Vic; el de Pau Vila, sobre la Cerdanya.

En el año 1931, la "Casa del Vallés", defensora de la división administrativa en comarcas organizó una serie de conferencias entre especialistas publicadas en El problema comarcal de Cataluña y que servirian de base y orientación a los trabajos de comarcalización llevados a cabo en la Segunda República.

\section{PROYECTO DE DIVISIÓN COMARCAL}

Pau Vila en su estudio presenta los caminos seguidos para llegar a establecer, con unos criterios fundados, una división de Cataluña en co- 
marcas, naturalmente con la supresión provincial, impuesta por el liberalismo del siglo pasado.

Procederían en primer lugar a conocer los criterios seguidos por los diferentes tratadistas, en las divisiones comarcales que habian establecido; los libros escolares de Flos i Calçat y Pere Blasi, el primero con 46 comarcas, el segundo con 38; el periodista Pagés y Rueda, defensor de la concentración comarcal agrupaba las comarcas secundarias, poco extensas. Pero los criterios por ellos seguidos, según Pau Vila, eran puramente nominalistas.

Los científicos Costa (botánico) y Faura (geólogo) encuentran grandes dificultades en la delimitación. El resultado para Costa, es la gran desproporción de tamaño, gigantescas como las de alta Montaña, reducidas como las de la Vega.

Las divisiones de Aulestia i Pijoan y Sunyol se asemejan en los criterios fisiográficos establecidos. Mossen Font i Sagué el primero que realizó un estudio particular de cada comarca en la que estudia la geografía, orografía, hidrografía, etnografía, historia, con consulta de los archivos provinciales, resultándole muy difícil establecer una división comarcal.

Se procederá a conocer lo que la historia contemporánea ha aportado en las delimitaciones comarcales, con los problemas de nomenclatura, pues en los documentos hay comarcas que aparecen con diferentes nombres. Ante estos problemas, consideran que, a la hora de elegir, 10 mejor es proceder a la elección de la comarca natural, que a escala menor repite los rasgos generales de la región geográfica dentro de la que está encuadrada «las comarcas no son más que matizaciones dentro del conjunto general, y de estas características procede la vaguedad de sus límites".

A la hora de establecer las comarcas se planteaba el nombre de la nomenclatura, el seleccionar los nombres de las comarcas que respondian a una necesidad viva, con las que el pueblo se identificaba, tales como al Ampurdán, el Vallés, la Selva, Urgel, etc. Pero estos territorios, como se ha expuesto en los problemas de delimitación, estaban rodeadas de unas zonas, en las que los caracteres se iban desdibujando zonas de transición y de cambios. Así los responsables de la división comarcal de Cataluña, tuvieron que proceder a seleccionar los nombres, y proceder a una agrupación en categorías. En primer lugar, seleccionaron a las de origen histórico, unas de muy vieja tradición como el Ampurdán, Segarra, Cerdaña, otras de origen medieval, de tipo señorial 
como los condados de Urgel, Pallars, Ribagorza, otras monásticas como el Priorato.

En segunda categoría, las derivadas de un núcleo urbano, tales como el Moianes, Bergueda, Plana de Vich, campo de Tarragona, Barcelonés.

La tercera categoría derivada de aspectos fisiográficos tales como la Maresma, Costa de Levante, Vallés. visiones.

Partiendo de estas tres categorías podrían llegar a establecer subdi-

\section{MÉTODO DE TRABAJO Y POSTERIOR APLICACIÓN POLITICO- ADMINISTRATIVA}

Los expertos encargados de establecer esta ordenación del territorio catalán tuvieron conciencia de que era una dura tarea, no sólo recorrer el terreno, hablar con las gentes, encuestas, sino el recoger bibliografía, consultar los archivos, interpretar la toponímia, etc.

Sin olvidar la valoración de la realidad presente, los aspectos económicos, las relaciones sociales.

El propósito de este trabajo, con la ordenación comarcal era el borrar las provincias, por considerar que estas habian traído un desequilibrio social y económico en el espacio catalán.

Un trabajo minucioso científico llevaría largo tiempo, y como dice Pau Vila, uno de los encargados de esta tarea coincidiendo con las palabras del historiador Valle i Taberner: "En lo posible se han de inscribir fenómenos nuevos en los cuadros antiguos con las modificaciones que en ellos quepan".

Habría que reducir en lo posible el número de comarcas «con el fin de relacionar los pueblos más alejados con la capital. Estudiando los medios de comunicación, teniendo en cuenta que los centros comarcales, no sólo serían centros administrativos, sino también hogares culturales, proyectados hacia las tierras altas. No se pasaría de una treintena de comarcas a ser posible menos. Las comarcas pequeñas se aglutinarían y las grandes se dividirían» (Vila, obra citada, 1977, p. 97). Habría que estudiar con cuidado los centros de mercado y las comunicaciones, y 
hacia donde se dirigen las gentes de la comarca. "Este estudio servirá para el control de la capital de la comarca, pudiendo ser necesario el escoger otra capital sin la actividad comercial de mercado. La capital no tiene porqué ser inamovible, pudiendo cambiarse, si fuere necesario".

Teniendo en cuenta lo expuesto Pau Vila, en 1930, presentó el proyecto en 1930 de las siguientes comarcas:

El Ampurdán, dividido en dos, el Alto Ampurdán con la capital en Figueras y el Bajo Ampurdán en San Feliú de Guixols; la Selva a la que incorporaba el Gironés, y la "rodalia" de Bañolas, dadas sus relaciones comerciales y sociales, con Gerona. La comarca de la Garrotxa, con la capital en Olot, por ser centro agrícola, mercantil e industrial de gran atracción. La Cerdaña y el Ripollés, considerando la capital en Ripoll, aunque en Puigcerdá por su posición fronteriza tendría ciertos organismos oficiales; Osona, con la capital en Vich; el Bergueda, con Berga; el Cardoner y el Begés; la cuenca de Odena; el Vallés; la Maresma, el Barcelonés; el bajo Llobregat; la Segarra; la Noguera; Urgel, dividido en Alto, Medio y Bajo; el Pallars, dividido en dos, Pallars Jussá y Pallaro Sobirá; el valle de Arán.

Esta propuesta de división y estudio de Pau Vila no se llevó a cabo en el año 1936.

\section{LA DIVISIÓN TERRITORIAL DE CATALUÑA}

Al establecerse la República y preparada la autonomía de Cataluña, era necesaria una división territorial, pues se consideraba «que la división provincial había producido una herida que con el despertar de la sensibilidad política se había hecho viva; por eso hoy, restablecida la unidad catalana, no se quiere tener rastro en la futura reorganización administrativa, pues hay que recordar que los territorios provinciales presentan anomalías de atribución, como la de incluir el Solsonés en Lérida y el Ripollés en Gerona (Vila, obra citada, p. 123).

Por esta razón se propuso una ponencia para su estudio, de la que tomaron parte los geógrafos Pedro Blasi, José Iglesies, Miguel Santaló, Felipe Solé, Pau Vila y el historiador Rovira y Virgili.

Se fijaron unos principios orientadores, $1^{\circ}$, que el número de comarcas no fuese muy elevado; $2 .^{\circ}$, que cada pueblo de una demarcación 
pudiese ir en un dia a pie al centro comarcal; $3 .^{\circ}$, que las demarcaciones tuviesen un cierto equilibrio en el número de habitantes $O$ al menos que la extensión compensase la diferencia; $4 .^{\circ}$, que como había poblaciones que eran cabeceras en la división político-administrativa vigente con organismos oficiales se procuraría descargarles del trabajo que les pudiese ocasionar las nuevas demarcaciones.

La ponencia gozó de una gran libertad de acción al no recibir directrices del gobierno y no estar aprobado aún el Estatuto.

El primer paso sería el documentarse sobre el concepto popular de las comarcas; fijar las zonas de mercados para establecer las demarcaciones económicas, y hacer una crítica de la existencia de los partidos judiciales en relación con los aspectos económicos y sociales.

Fijado el plan se procedió a enviar a todos los Ayuntamientos de Cataluña un cuestionario sencillo en el que se formulaban tres preguntas, ¿a qué comarca pertenece vuestro pueblo?, ¿a qué lugar de mercado van principalmente?, ¿si van a algún otro mercado?

Respecto a los partidos judiciales se vio que no estaban mal estructurados, pues la mayor parte, su capital, era centro comarcal y de mercado.

El resultado de las encuestas era caótico, aparecian más de 200 nombres de comarcas, con una diferencia en el número, oscilando entre 19 y 50 . El mapa hecho según la encuesta, no servía de base de trabajo para la ponencia, lo que no sucedía con los mercados, que coincidian aproximadamente con las cabezas de partido. No obstante, al esbozar la ponencia en 1932 un ante-proyecto de las demarcaciones fijaban el número de 28 lo que era una reducción frente a los 36 partidos judiciales existentes.

En este proyecto, dice Pau Vila, "las divisiones que se proponían, por el hecho de ser solo 28 eran demasiado extensas y cabalgaban algunas sobre ciertas comarcas y obligaban a dejar desatendidos centros de mercado o núcleos importantes verdaderas cabeceras comarcales. Pero, tratándose de una estructuración de primer grado, por la cual cada unidad territorial se debia de relacionar directamente con Barcelona era conveniente reducir lo más posible el número de demarcaciones, para no prodigar en exceso los servicios de gobierno" (Vila, obra citada, p. 133 y 134).

Este proyecto, en el que se habla de "demarcaciones" y no de comarcas, reflejaba una gran artifiosidad, al querer huir de las comarcas que a pesar de su imprecisión constituyen una realidad viva. El problema 
era que pudiesen ser más numerosas que las demarcaciones, que si obligaba a un elevado número de organismos oficiales se podía evitar, agrupando comarcas con las que se formarian supracomarcas.

En el segundo proyecto, aprobado el 12 de octubre en sesiones presidida por el Consejero de Gobernación José Tarradellas, Cataluña quedaba dividida en 39 comarcas ordenadas en nueve agrupaciones a las que se dio el nombre de "Veguerias".

La Veguería de Gerona, con 182 municipios y las comarcas de Alto y Bajo Ampurdán, Gironés y la Selva.

La Vegueria de Barcelona con 125 municipios y las comarcas de la Maresma, Vallés, Oriental y Occidental, Barcelonés y Bajo Llobregat.

La Veguería de Tarragona con 93 municipios y las comarcas de Garraf, Alto y Bajo Panadés, Tarragonés y Alto Campo.

La Veguería de Reus con 91 municipios y las comarcas del Bajo Campo, Cuenca de Barberá, Priorato y Ribera.

La Veguería de Tortosa con 33 municipios, el Bajo Ebro, Montsiá y Tierra Alta.

La Vegueria de Vich con 127 municipios y las comarcas de la Garrotxa, Osona, Ripollés y Cerdaña.

La Veguería de Manresa con 116 municipios y las comarcas del Bages, Berguedá, Anoia y Solsonés.

La Veguería de Lérida con 165 municipios y las comarcas de Segarra, Urgel, Garrigas, Segriá y Noguera.

La Veguería de Seu-Tremp con 129 municipios y las comarcas de Alto Urgel, Pallars-Jussa, Pallarós Sobira y Valle de Arán.

Este proyecto fue publicado ${ }^{4}$, pero se demoró su presentación en el Parlamento Catalán, aunque se suprimieron los gobernadores civiles, sin haberse suprimido las provincias.

En el mes de agosto de 1936, al crearse el Consejo de Economía, se sintió la necesidad de ordenar el territorio de la Generalidad, bajo el aspecto comarcal. Una nueva ponencia estudiaba el proyecto de las demarcaciones comarcales que no era otro que el del período anterior, con

${ }^{4}$ Divisió Territorial. Estudio i proyectes. Nomenclator de municipios, 370 p. y 4 mapas 1: 630.000 . 
algunas rectificaciones. Se cambiaba el nombre de veguerias por el de regiones y se alteraban algunas comarcas.

\section{GEOGRAFÍA Y COMARCAS}

La organización espacial del período republicano, en sus proyectos y tardía aprobación, quedó en suspenso en 1939, restableciéndose la división provincial, con los correspondientes partidos judiciales.

En la década de los 70 , un equipo de geógrafos catalanes bajo la dirección de Solé Sabaris, publicarían la Geografía de Catalunya, en tres volúmenes. El primer volumen dedicado a la Geografia general, fisica y humana y los otros dos a la Geografía comarcal. Se inicia esta con una Introducción dedicada a la Comarca como unidad natural. Se dice que las comarcas corresponden a las células fundamentales del tejido o estructura geográfica.

Para definir y delimitar una comarca se han de buscar los elementos naturales, relieve, clima, vegetación, que la caracterizan y definen su unidad, pero la comarca es una síntesis de elementos físicos y humanos. La presencia humana también dibuja y delimita unos sectores. La población, su distribución y composición, así como las actividades económicas, factores espirituales, formas de vida, imprimen un sello al espacio. El hombre económico es un gran diversificador de los espacios, a la par que, con el dinamismo de sus actividades, da movilidad en el marco comarcal.

Ahora bien, para establecer la división comarcal, no es suficiente el tener en cuenta la síntesis de factores naturales y humanos; factores no geográficos sino administrativos y políticos han dado nombre a determinadas comarcas. Dicen los autores que, aún existiendo entre el pueblo una clara conciencia comarcal, a la hora de establecer una división, con unos nombres precisos, se presenta la confusión, tal como se ha expuesto, sucedió con la Ponencia para la división territorial.

Asi pues, en el momento de establecer un mapa comarcal se ha de tener presente que la comarca es una síntesis, en la que se combinan elementos naturales y elementos humanos. Son estos últimos los creadores del paisaje, y para su establecimiento los autores han procedido a situar las comarcas dentro de las grandes unidades regionales de Cata- 
luña. Han concretado todas las características físicas que le dan unidad. Dentro del aspecto social han procedido a un estudio de la población, ocupación del suelo, evolución del efectivo humano, distribución y densidades actuales. Las actividades económicas ocupan el primer plano en la exposición comarcal, insistiendo en la rama dominante con repercusiones en los aspectos sociales y paisajísticos, con la influencia decisiva en la comarca de los centros o capitales comarcales.

La delimitación y la nomenclatura plantean problemas unos por la dificultad a la hora de establecer los límites, dado el carácter de concentración hacia el centro de los caracteres que establecen la unidad, y la debilitación de los mismos hacia la periferia, hacia las zonas de transición.

En la geografía a la que hago referencia se presentan las comarcas dentro de las grandes unidades regionales en las que han dividido Cataluña. La región del Pirineo y Prepirineo ${ }^{5}$, con las comarcas del Valle de Arán, Ribagorza, Pallars, Andorra, Alto Urgel, Berguedá, Cerdaña.

La Cataluña ultrapirinaica ${ }^{6}$, con las comarcas de El Capcir, El Conflent y El Vallespir.

Región de la Tramuntana ${ }^{7}$, con las comarcas del Rosellón y el Ampurdán.

Región de la Montaña Oriental húmeda ${ }^{8}$, con las comarcas del Ripollés, Osona, Garrotxa, el Gironés, la Selva.

La Región del Altiplano Central ${ }^{9}$; con las comarcas de Solsonés, Segarra y Bages.

La Región Continental ${ }^{10}$, con las comarcas de Urgel, La Garriga, La Noguera, Segriá, Llitera, Bajo Cinca, Tierra Alta y Matarraña.

La Región mediterránea ", con las comarcas del Bajo Ebro y Montorio, Priorato, Ribera del Ebro, Campo de Tarragona, Cuenca de Barberá, Panadés y Garraf, Anoia, El Vallés, la Maresma, el Bajo Llobregat y el Barcelonés.

${ }^{5}$ Con los estudios de Solé Sabaris, Salvador Lloret, Rafael de la Torre, Juan Rabagliato, Pau Vila.

- Francisco Doumenge, Roger Lambert, G. Cuillé, R. Vinás.

7 Solé Sabaris, F. Doumenge, Alberto Compte.

- Solé Sabaris, Llobet, Maria Bolós.

9 Juan Vilá, Manuel Riu, Juan Rebagliato.

10 Solé, Renat Perrin, José Tortosa, F. Pita, Rabagliato, G. Gimeno.

" José Iglesies, Utmillen, G. Giralt Llobet, Rabagliato. 


\section{LA ORGANIZACIÓN ACTUAL DEL ESPACIO CATALÁN}

La organización territorial ha sufrido profundas transformaciones desde el primer tercio del siglo actual. El desarrollo industrial, el aumento de espacios residenciales que sobrepasan los límites municipales, la expansión de Barcelona y su área metropolitana. Tradicionales cabezas comarcales en decadencia, municipios abandonados, crecimiento de nuevos núcleos urbanos, han alterado la tradicional red urbana comarcal.

Actualmente en la organización territorial de Cataluña destaca el papel de Barcelona como centro motor, con su área metropolitana dando cohesión y unidad a todo el territorio. Barcelona se comporta como centro aglutinador de cinco áreas: el área metropolitana de Barcelona y las que se estructuran entorno a Gerona, Lérida, Manresa, Tarragona y Reus.

Las tres capitales de provincia ejercen de centros ordenadores. Dentro de estas grandes áreas regionales hay otros centros urbanos menores, Tortosa, Vich, Figueras, que sirven como centros comarcales, otras menores, como Igualada, Villafranca del Panadés, Sabadell, Tarrasa, etc., otras de carácter histórico que conservan cierta unidad como el Moianes, Lluçanes, y otras que carecen de vinculación a un centro concreto, que dependen de centros regionales como Pobla de Segur.

El 4 de abril de 1987 el parlamento catalán aprobó leyes referentes a la organización territorial.

$1 .^{\circ}$ Supresión de las Diputaciones provinciales, pasando sus competencias a la Generalidad, con el fin de convertir al Principado en una Comunidad Autónoma uniprovincial.

2. Definición de la Comarca como elemento potenciador de la capacidad de gestión de los municipios. Por el momento se aceptan las 38 comarcas de 1936, pero se estudia una nueva restructuración. Las competencias de las comarcas son: ordenación del Territorio y urbanismo, sanidad, servicios sociales, cultura, deportes, salud pública y medio ambiente.

3. Supresión de la Corporación Metropolitana de Barcelona, creada en 1974 y estructuración de dos nuevas entidades metropolitanas, una la de Transportes y otra de servicios hidráulicos y tratamiento de residuos. 
La originalidad de este plan de ordenación del territorio, parece ser la de potenciar la municipalidad, como agrupación de municipios, en donde se gobiernen y gestionen las competencias de la administración local y, por delegación, muchas de las competencias de la Generalidad. 


\section{DIVISIÓN TERRITORIAL DE CATALUÑA (1936)}

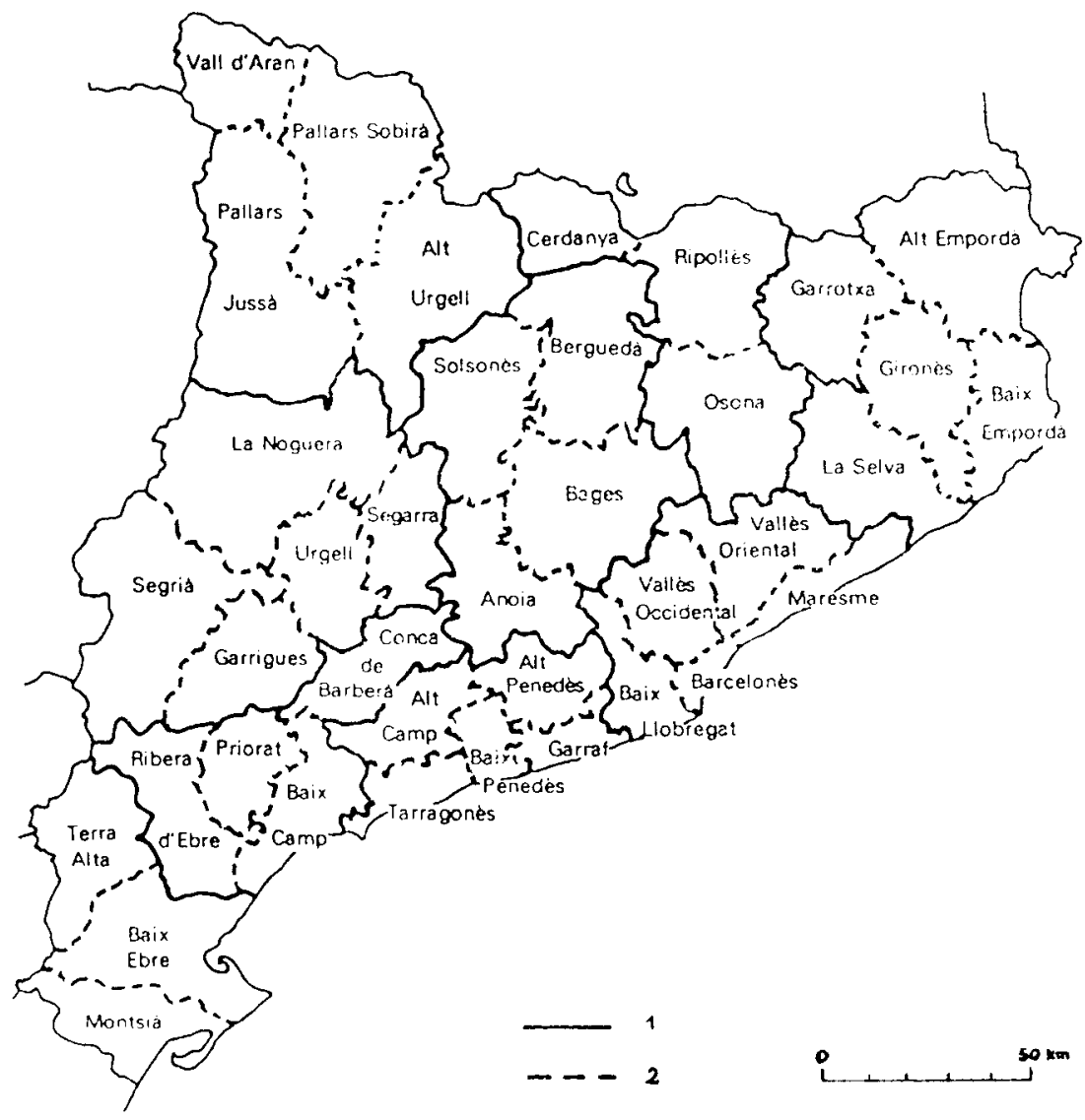

1. Límite de las regiones o veguerías.

2. Limite de comarca.

Fuente: Geografía de Catalunya, dirigida por Solé Sabaris (1974). 


\section{BIBLIOGRAFÍA}

ALBET, M., 1975: “Pau Vila, una vida plena». Miscelánea Pau Vila, Barcelona.

CASAssas i Simo, LI., 1974: “Notes per l'estudi del pensament geográfic a Catalunya". Homenaje a M. $^{a}$ de los Angeles Ferrer Sensat, Barcelona.

-1979: «Unes pàgines de la história del pensament geográfic a Catalunya". Butlletí de la Institució Catalana d'Historia Natural, n. ${ }^{\circ} 43$, Barcelona.

-1988: «El debate de la organización territorial de Cataluña. ¿Hacia el reparto de las minucias del poder?». Rev. Ería, n. ${ }^{\circ} 15$, p. 15-26.

Deffontaines, P., 1978: Geografia de los països catalans. Ed. Ariel, Societat Catalana de Geografía.

Galera, M., 1975: “Bibliografía de Pau Vila». Miscelanea Pau Vila, Barcelona.

IGLESIES, J., 1975: Presencia de l'excursionisme dins la cultura catalana. L'excursionisme a Catalunya, 1876-1975. Barcelona. Fundación Carulla-Font.

Lorman, J.; Planas, I., 1987: Geografía de Catalunya. Barcelona, Ed. Claret.

-: Revista Catalana de Geografía, número especial dedicado a Josep Iglèsies; enero, febrero y marzo, 1978.

Solé SABARIS, L. (Dir.), 1974: Geografía de Catalunya, 3 vols. Barcelona. Ed. Aedos. 
SOlE SABARIS, L., 1974: "L'ensenyament de les ciències naturals a Catalunya mig segle enrera", en Homenaje a M. ${ }^{a}$ de los Angeles Ferrer Sensat. Barcelona.

-1975: “Los primeros geólogos catalanes". Revista de Estudios Geológicos, vol. 31. Madrid, CSIC.

VILA, P., 1977: La divisió territorial de Catalunya (Selecció d'escrits de Geografia). Barcelona, Ed. Curial.

-1978: Aspects geográfics de Catalunya. Barcelona, Ed. Curial.

-1979: Opinions d'un geófraf i actituds d'un ciudadá. Barcelona, Ed. Curial.

VILA, P., y CASASSAS, LI., 1974: Barcelona i la seva rodalia, al marg des temps. Barcelona, Ed. Aedos. 